\title{
Sling effect in AA-interactions and its implication for UHECR
}

\author{
A.D.Erlykin ${ }^{\mathrm{a}} *$, A.W.Wolfendale ${ }^{\mathrm{b}}$ \\ ${ }^{a}$ P.N.Lebedev Physical Institute Leninsky prosp. 53, Moscow 119991, Russia \\ bPhysics Department, University of Durham, South Road, Durham DH1 3LE, UK
}

The 'Sling effect' appears when a fragment of a projectile nucleus emitted after a peripheral collision of the latter with a target nucleus is caused to rotate with a high spin. The spinning fragment has a deformed shape and looks like an oblate ellipsoid. Due to the virtual non-compressibility of nuclear matter and the polarization of the spin in the plane transverse to the input momentum of the projectile nucleus such an ellipsoid has a reduced mean interaction cross-section compared with a non-spinning fragment which has a spherical shape. Due to purely geometrical arguments, such an ellipsoidal nucleus should have additional fluctuations of the cross-section even at the fixed impact parameter dependent on the orientation angle between the axis of the ellipsoid and the vector connecting centers of projectile and target nucleus. The number of wounded nucleons in the projectile nucleus participating in the interaction correlates strongly with the interaction cross-section. All these reasons lead to a non-exponential attenuation of fragments and an increased probability of finding the fragment at a large depth in the absorber.

If the sling effect appears in the interaction of a primary cosmic ray nucleus with nuclei in the atmosphere the induced atmospheric cascade will have a slower attenuation, and thereby can help to reduce some inconsistencies in the interpretation of the existing experimental data on extensive air showers, observed in the lower half of the atmosphere. It will also have an implication on the development of nucleus-induced cascades at ultra high energies.

\section{Introduction}

Information about nuclear interactions at energies higher than $2 \mathrm{PeV}$ comes only from cosmic rays, viz. from the study of atmospheric cascades initiated by primary cosmic ray protons and nuclei. Due to the unknown mass composition of the primary cosmic rays the only criterium of the correctness of the chosen interaction model, the mass composition and the adopted method of analysis, is the consistency of the results on the primary mass composition derived from different observables. The 'big leap' forward in this direction has been made with the development of the CORSIKA code for the simulation of extensive air showers ( EAS ) which can incorporate and test different interaction models [1]. Using the results obtained with CORSIKA it was shown that the QGSJET model gives in general the most consistent description of the observed EAS characteris-

*E-mail: erlykin@sci.lebedev.ru tics and the mass composition 23 .

However, the deeper analysis of the EAS data collected by the KASCADE experiment reveals that some inconsistencies still remain [45. There is a difference in the mass composition derived from observables which either include or ignore $N_{e}$ - the electron size of the shower, as well as between results obtained from the ground-based measurements and from the distribution of $X_{\max }$ - the depth of the shower maximum in the atmosphere measured by means of Cherenkov light, etc. There are different attempts to overcome these inconsistencies 67. All of them agree that improved interaction models should make the atmospheric cascade more penetrative and propose different mechanisms for that. We also examined this problem and came to the conclusion that the best way is to invoke a minor increase of the energy fraction transferred to the electromagnetic component $\left(\mathrm{K}_{\gamma}=0.26\right)$ with an increase of the 'elongation rate'(the change in depth of maximum 
per decade of energy); the value needed is about $71 \mathrm{gcm}^{-2}$. Both modifications are easier to associate with nucleus-nucleus ( AA ) interactions [8]. In this paper we proceed further in this direction.

\section{Sling effect}

\subsection{Fragmentation of the high energy nu- cleus}

There is a vast literature on different aspects of AA - interactions. Important for our subject is the fact that the fragmentation of a projectile nucleus does not vanish at relativistic energies i.e. the nucleus is not split into independent nucleons. On the contrary, the yield of heavy fragments rises with energy up to 200 $\mathrm{GeV} /$ nucleon due to an increasing role of an electromagnetic excitation and dissociation of projectile nuclei [9]. For instance, in the interactions of Fe group nuclei $(22<Z<28)$ with nuclear emulsion at $20-65 \mathrm{GeV} /$ nucleon such a heavy fragment as $\operatorname{Sc}(Z=21)$ has been observed, among others [10. Electromagnetic nuclear excitation and multifragmentation is observed in ultra-peripheral collisions even at RHIC energies $(\sqrt{s}=200 \mathrm{GeV} /$ nucleon, which corresponds to an equivalent energy of $4.3 \mathrm{PeV}$ for gold in the laboratory coordinate system [11 ) and used as a collider luminosity monitor [12. In what follows we postulate that the process of nuclear fragmentation persists up to $\mathrm{PeV}$ energies in AAinteractions.

\subsection{Rotation, polarization and deforma- tion of the fragment}

Nuclear fragments arising in grazing peripheral collisions at sub-GeV energies often rotate like a 'sling' and achieve a high spin. This spin is polarized in the plane transverse to the momentum of the projectile nucleus. The effect is used at low and intermediate energies for the formation of polarized beams of low intensity [13. Inspite of the fact that the nuclear fragment is a composite, and often excited object, it demonstates the features of the collective motion and behaves like a liquid drop. Some features of collective motion of the nuclear matter, viz. the elliptic flow, are preserved even at relativistic energies [11. In this connection we assume that the features of the fragment's rotation and polarization persist up to $\mathrm{PeV}$ energies.

Due to the rotation of the object with liquid drop properties its shape is deformed. The spherical fragment becomes an oblate ellipsoid with a shorter axis coincident with the axis of the spin. Following [14] we call the effect of the rotation, polarization and deformation of a nuclear fragment as the 'sling effect'. In the experimental study of deformed nuclei, spins of up to a few tens and deformations of the nuclear sizes up to $\sim 30 \%$ have been observed.

\section{A geometrical approach}

\subsection{General remarks}

Many features of AA-interactions are considered within a pure geometrical approach. The popular Bradt-Peters formula for the interaction cross-section of two nuclei [15] is just the result of the geometrical examination of the collision between two spheroids. The formulae for the calculation of the mean number of wounded nucleons in colliding nuclei were also derived using geometrical arguments [1617. We continue our consideration using the same geometrical approach. As an example, we consider the collision of the iron nucleus ${ }^{56} \mathrm{Fe}$, which is one of major constituents of cosmic rays, with a nitrogen nucleus ${ }^{14} \mathrm{~N}$ of the Earth's atmosphere. As a result of this collision the fragment ${ }^{45} \mathrm{Sc}$ emerges and the remaining 11 nucleons are liberated from the parent iron nucleus with, on average, 8 being 'wounded'.

\subsection{The reduction of the mean cross- section}

We assume that the Sc-fragment moves down in the vertical direction and is subject to the 'sling effect'. This means that it rotates and has an ellipsoidal shape with a shorter axis orientated in the horizontal direction. Let us denote its longer axis as ' $a$ ' and the shorter axis as ' $c$ '. The deformation of the spheroid is often characterized by an ellipticity $\epsilon$ which is defined as $\epsilon=\sqrt{1-c^{2} / a^{2}}$. Due to the non-compressibility of nuclear matter the volume of the deformed fragment should be equal to the volume of the 
spherical one, i.e. $\frac{4}{3} \pi a^{2} c=\frac{4}{3} \pi R^{3}$, where $R$ is the radius of the spherical fragment. Combining the formulae for $\epsilon$ and for the volume we obtain $a=R /\left(1-\epsilon^{2}\right)^{1 / 6}, c=R\left(1-\epsilon^{2}\right)^{1 / 3}$. If the ellipsoidal fragment moves down and its shorter axis is orientated horizontally then its geometrical crosssection seen 'edge-on' from below is $\pi a c$. It means that this cross-section is equal to $\pi R^{2}\left(1-\epsilon^{2}\right)^{1 / 6}$, i.e. reduced compared with a spherical fragment by the same factor of $\left(1-\epsilon^{2}\right)^{1 / 6}$ as the increase of the larger axis.

In what follows we examine two cases with $\epsilon=0.5$ and 0.9 . The latter one corresponds to the increase of the larger axis by about $32 \%$ and to the reduction of the shorter axis by $43 \%$, which approximately corresponds to the magnitude of the deformation observed in experiments hitherto. For these values of $\epsilon$ the reduction of the purely geometrical cross-section is $5 \%$ and $24 \%$ respectively, which definitely should reduce the mean interaction cross-section between this fragment and the target nitrogen nucleus.

In order to estimate this reduction we introduce the so called collisional cross-section, i.e. the cross-section for a single collision. It is equal to the overlap area between two colliding objects ( Figure 1 ). In the case of the two spherical objects it depends just on the radii of the objects $R, r$ and the impact parameter $b$. In the case of the colliding ellipsoid and spheroid the overlap area depends not only on $a, c, r$ and $b$, but also on the orientation angle $\alpha$ between the longer axis of the ellipsoid and the vector connecting the centers of the two objects ( see Figure 1 ). We have calculated this dependence for colliding ${ }^{45} \mathrm{Sc}$ and ${ }^{14} \mathrm{~N}$ nuclei with $\epsilon=0,0.5$ and 0.9 . The result of the integration of the overlap area over the impact parameter $b$ as a function of the orientation angle $\alpha$ is shown in Figure 2. For simplicity the radii of non-deformed $\mathrm{Sc}$ and $\mathrm{N}$ nuclei have been taken as $R, f m=1.2 \cdot A^{1 / 3}$, where $\mathrm{A}$ is the mass of the nucleus and the interaction occured in every case when the overlap area was non-zero. It is seen that the dependence of the cross-section on $\alpha$ and the reduction of its mean value compared with the case of the non-deformed fragment with $\epsilon=0$ becomes stronger with an increase in ellipticity.
COLLISION OF SPHERICAL AND ELLIPSOIDAL NUCLEI

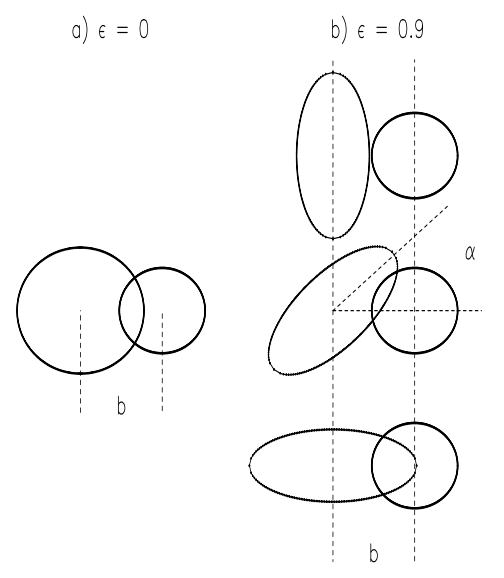

Figure 1. A schematic view of the collision: a) between two spherical nuclei and $b$ ) between an ellipsoidal fragment $(\epsilon=0)$ and spherical target, the impact parameter being the same in the two cases. It is seen that the overlap area in the latter case depends on the orientation angle $\alpha$ between the larger axis of the ellipsoid and the vector connecting centers of two nuclei even for a fixed impact parameter $b$.

\subsection{The attenuation of the deformed frag- ment}

When the collisional cross section fluctuates, the interaction rate of the fragment changes and becomes non-exponential. We calculate it as

$\frac{d P}{d z}=\int_{0}^{\infty} \sigma \exp (-\sigma z) \frac{d P}{d \sigma} d \sigma$

where $P$ is the probability for either the fragment to interact at the depth $z$ in the expression for $\frac{d P}{d z}$ or to collide with a cross-section $\sigma$ in the expression for $\frac{d P}{d \sigma}$. We used a gaussian approximation of the $\sigma(\alpha)$ function in the range of $\alpha=0^{\circ}-90^{\circ}$ and the result of the integration compared with the interaction rate for the spherical fragment is shown in Figure 3. The non-exponential character of the interaction rate increases with an ellipticity. It is seen that interaction rate of fragments 


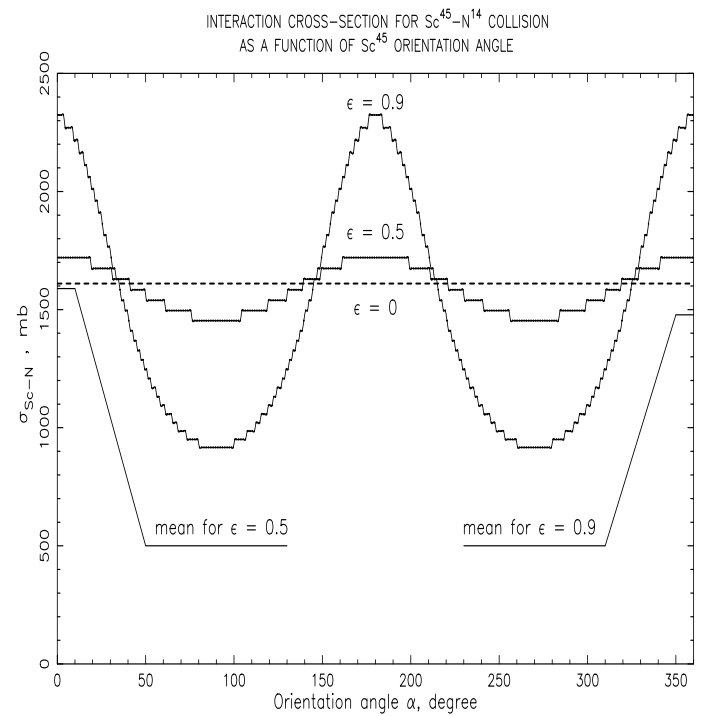

Figure 2. The interaction cross-section for ${ }^{45} \mathrm{Sc}-{ }^{14} \mathrm{~N}$ collision as a function of ${ }^{45} \mathrm{Sc}$ orientation angle $\alpha$ for the ellipticity $\epsilon=0$ (dashed line), 0.5 and 0.9 (indicated in the Figure ). The mean values of the cross-section averaged over the uniform distribution of $\alpha$ are also indicated for $\epsilon=0.5$ and 0.9 . The reduction of the mean cross-section for a deformed fragment of Sc with respect to a spherical one with $\epsilon=0$ is clearly seen.

changes with atmospheric depth. The reduced rate at small depths leads to a higher probability for the fragment to penetrate deep into the atmosphere. It is the effect which we need in order to increase the penetrating ability of atmospheric cascades.

\subsection{The number of wounded nucleons}

The attenuation of the atmospheric cascade induced by a nucleus is determined not only by the interaction rate of the fragment, but also by the number of nucleons participating in the interaction, which are usually called 'wounded nucleons'. The meaning of the geometrical formulae used for the estimate of the mean number of wounded nucleons in the projectile nucleus $\mathbf{A}$ and in the tar-
RATIO OF INTERACTION RATES FOR DEFORMED AND SPHERICAL NUCLEAR FRAGMENTS

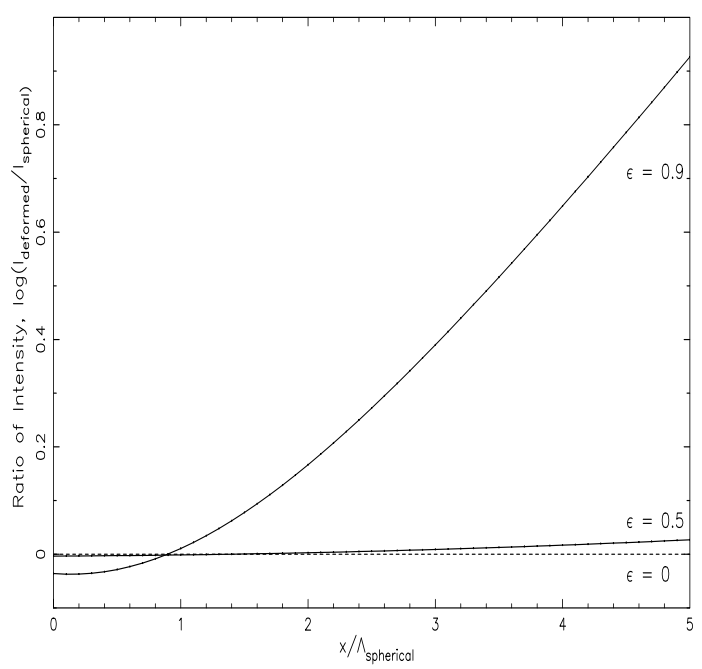

Figure 3. The ratio of the interaction rate for deformed ( $\epsilon=0.5$ and 0.9$)$ and spherical $(\epsilon=0)$ Sc fragments in the air. $\Lambda_{\text {spherical }}$ is the mean interaction length of the spherical Sc fragment in the air. The reduced interaction rate at small depths leads to a reduced attenuation and to a higher probability of finding the fragment deep in the atmosphere.

get nucleus $\mathbf{B}$ :

$$
\begin{aligned}
& n_{w}^{A}=A \frac{\sigma_{p B}}{\sigma_{A B}}=A \frac{S_{B} S_{A}}{\sigma_{A B} S_{A}}=A \frac{S_{\text {overlap }}}{S_{A}} \\
& n_{w}^{B}=B \frac{\sigma_{p A}}{\sigma_{A B}}=B \frac{S_{A} S_{B}}{\sigma_{A B} S_{B}}=B \frac{S_{\text {overlap }}}{S_{B}}
\end{aligned}
$$

is the mean number of nucleons in the $\mathbf{A}$ or $\mathbf{B}$ nucleus uniformly distributed over its geometrical cross-section, which is cut by an overlap area of colliding nuclei. We used this geometrical approach for the determination of the number of nucleons wounded in a single collision. In this case $S_{\text {overlap }}$ is the overlapping area which appears in one collision and can be different in another one. It depends on the ellipticity of the fragment $\epsilon$, impact parameter $b$ and orientation angle $\alpha$.

The dependence of $n_{w}$ on $\alpha$ for $\epsilon=0,0.5$ and 0.9 for different fixed impact parameters $b$ is shown in Figure 4. 


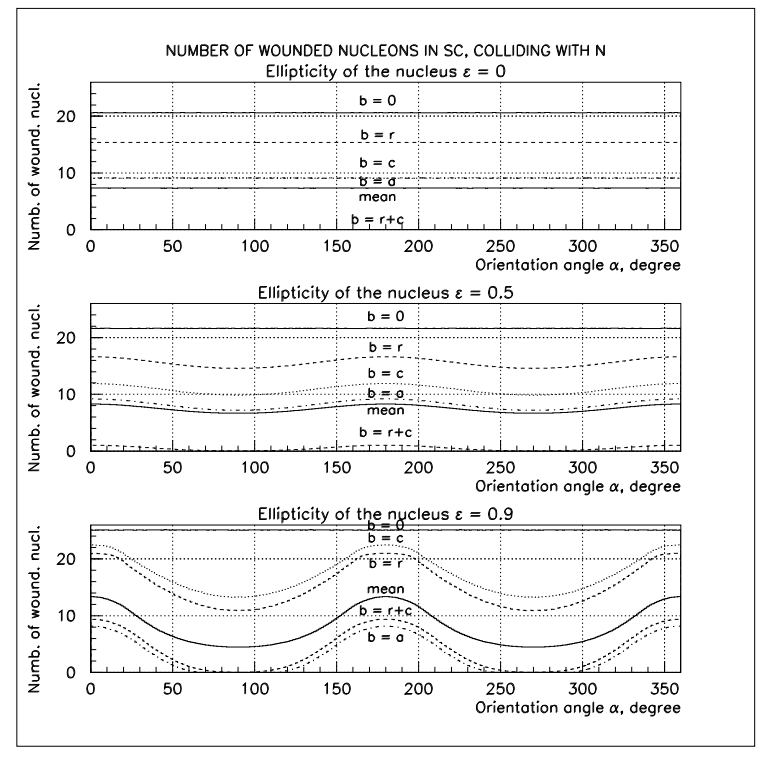

Figure 4. The dependence of the number of wounded nucleons $n_{w}$ in a single collision on the orientation angle $\alpha$ for the ellipticity of the projectile fragment $\epsilon=0,0.5$ and 0.9 and for the fixed impact parameters $b=0, r$ - the radius of target nucleus, $c$ and $a$ - small and large axes of the ellipsoid respectively, the sum $r+c$ and the mean $\left\langle n_{w}(\alpha)\right\rangle$ averaged over the impact parameter $b$. Values of impact parameters are indicated in the figure.

It is seen that the dependence on $\alpha$ increases with the ellipticity $\epsilon$. With an increased ellipticity the maximum impact parameter at which the interaction could occur increases too as well as the density of nucleons $A / S_{A}$ in the projectile nucleus. Due to this deformation the range of the impact parameters and the maximum number of wounded nucleons increase too ( Figure 5 ).

\subsection{The distribution of the number of wounded nucleons and its correlation with the cross-section}

Due to the stochastic fluctuations of the impact parameters and orientation angles the number of wounded nucleons also fluctuates. Its distribution is shown in Figure 6.

It is seen that the width of the distribution increases with the ellipticity. The important conse-

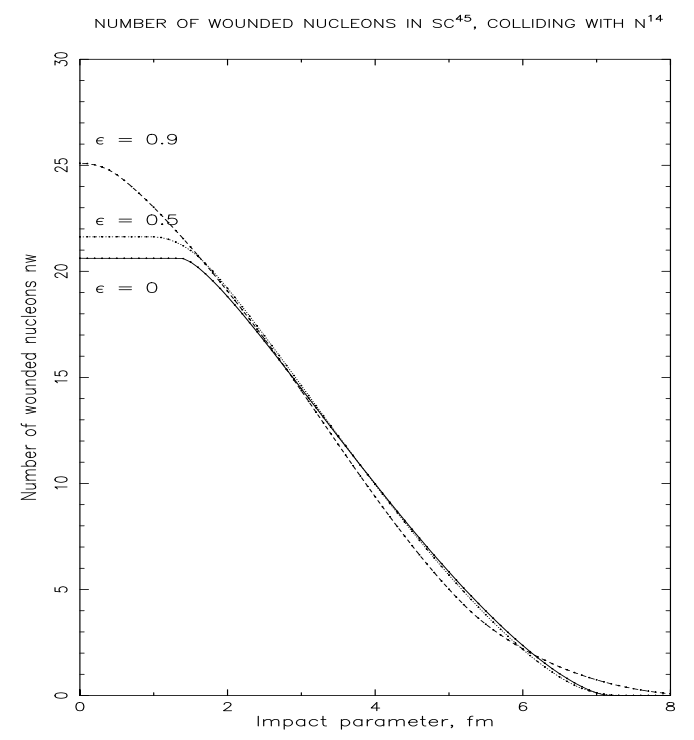

Figure 5. The dependence of the mean number of wounded nucleons $\left\langle n_{w}(b)\right\rangle$ on the impact parameter $b$ averaged over the uniform distribution of orientation angles $\alpha$.

quence of the deformation is the straightforward correlation between the number of wounded nucleons and the collisional cross-section. It is an evident feature because both $n_{w}$ and $\sigma$ are determined by the overlap area in the single collision. This correlation is shown in Figure 7 for $n_{w}$ and $\sigma$ averaged over all impact parameters. The existence of this correlation is a new feature for the interaction of deformed fragments and is important for their propagation through an absorber and the subsequent development of cascades initiated by high energy nuclei.

\section{The longitudinal development of the nucleus-induced cascade}

\subsection{The longitudinal development of the nucleon component}

In our example of the primary ${ }^{56} \mathrm{Fe}$ induced cascade we adopted the case where the interaction of the primary iron was normal with a deformed 


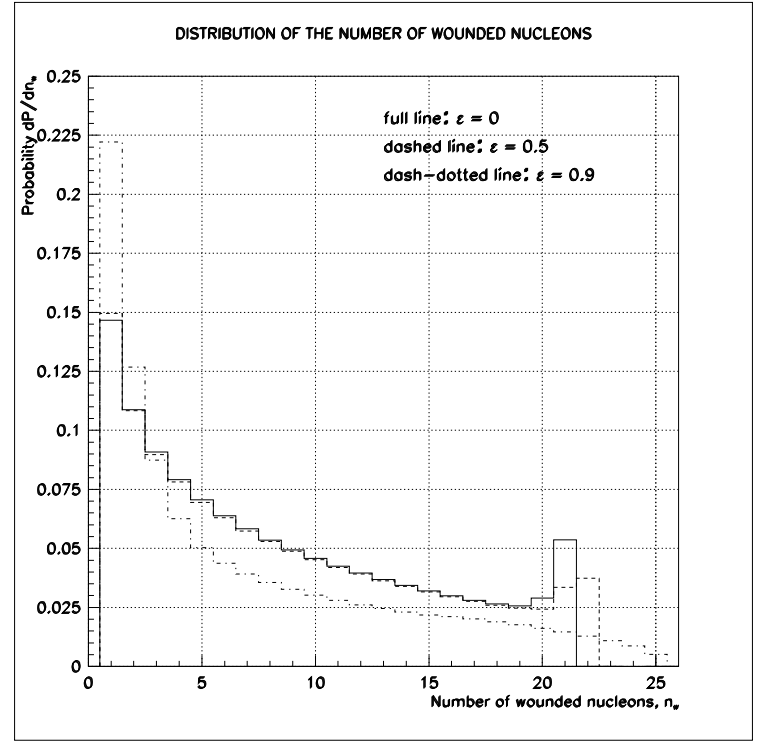

Figure 6 . The distribution of the number of wounded nucleons in ${ }^{45} S c-{ }^{14} \mathrm{~N}$ collision for the different ellipticity of ${ }^{45} \mathrm{Sc}: \epsilon=0$ ( full line ), 0.5 ( dashed line ) and 0.9 ( dash-dotted line ).

spinning ${ }^{45} \mathrm{Sc}$ fragment emitted. In the collision of the ${ }^{45} \mathrm{Sc}$ fragment with nitrogen nucleus of the air on average 7 projectile nucleons were wounded and the other 38 nucleons of the fragment liberated as spectators and propagated through the atmosphere by an ordinary way. Therefore the difference between the longitudinal development of the nucleon component in an ordinary cascade and the cascade with a spinning fragment is determined by the greater penetrating ability of the fragment. We have calculated the interaction rate of nucleons in our Fe-induced cascade with a spinning Sc fragment and compared it with the nucleon interaction rate in the ordinary Fe-induced cascade. The result for the case of $\epsilon=0.9$ is shown in Figure 8.

It is seen that in the cascade with a spinning fragment the interaction rate decreases at the initial stages of the cascade development and on the contrary increases later - the effect which we needed to make the cascade being able to pen-

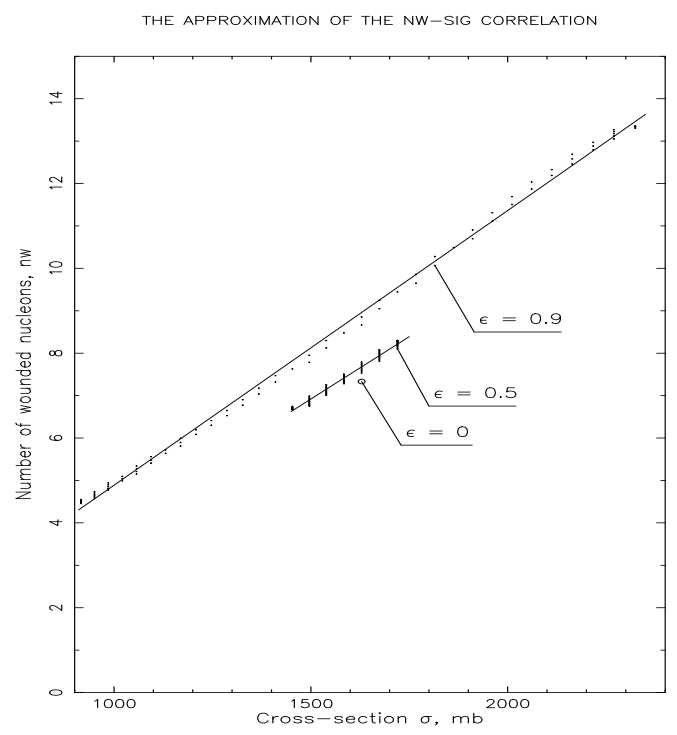

Figure 7. The correlation between the collisional crosssection and the number of wounded nucleons for the different ellipticity of the projectile fragment: $\epsilon=0,0.5$ and 0.9 .

etrate deeper into the atmosphere, although the absolute value of the effect does not exceed a few percent even for $\epsilon=0.9$. For $\epsilon=0.5$ it is even less.

\subsection{The longitudinal development of the Fe-induced cascade}

In order to calculate the influence of the sling effect on the development of the electromagnetic component of EAS we used the approximate formulae for the cascades initiated by nucleons proposed in [18 and used by us in [8. Interaction rates of nucleons in the Fe-induced cascade were taken from the previous subsection. The result for a primary Fe nucleus of $1 \mathrm{PeV}$ energy is shown in Figure 9. It is seen that the development of the electromagnetic component resembles that of nucleon component, viz. the cascade develops later, its size at the early stages of the development is smaller, but larger at large atmospheric depths. For the chosen values of ellipticity the magnitude 


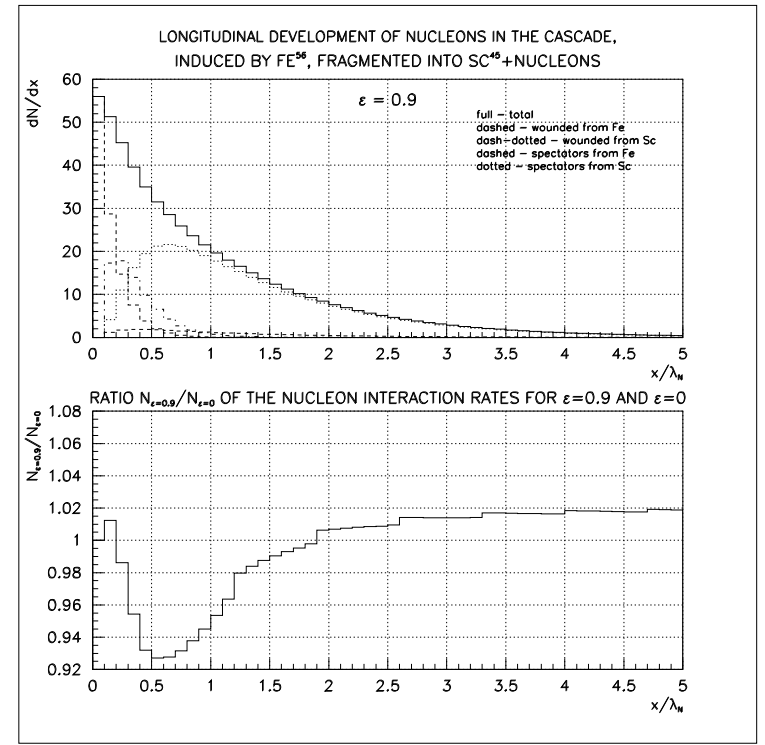

Figure 8. a) The interaction rate of nucleons in Feinduced cascade: dashed line - wounded nucleons from Fe, dash-dotted line - wounded nucleons from Sc, dashed line - spectators from Fe, dotted line - spectators from Sc, full line - total. b) The ratio of nucleon interaction rates in the cascade with a spinning Sc fragment and in an ordinary Fe-induced cascade with non-spinning Sc.

of the sling effect is small, however. The shift of $X_{\max }$ - the maximum cascade development, does not exceed $1 \mathrm{gcm}^{-2}$. The increase of the size at the sea level for $\epsilon=0.9$ is about $2 \%$. However the sling effect has the needed sign and it is definitely stronger for stronger deformations of the fragment.

\section{The implication of the sling effect for the ultra high energy cosmic rays}

At the moment it is difficult to make accurate estimates of the sling effect, because of lack of experimental data on the fragmentation of nuclei, excitation, polarization and interactions of the emitted nuclear fragments. In the examined example of the emission of just one ${ }^{45} \mathrm{Sc}$ fragment with the maximum ellipticity $\epsilon=0.9$ from the primary ${ }^{56} \mathrm{Fe}$ interaction the effect is shown to
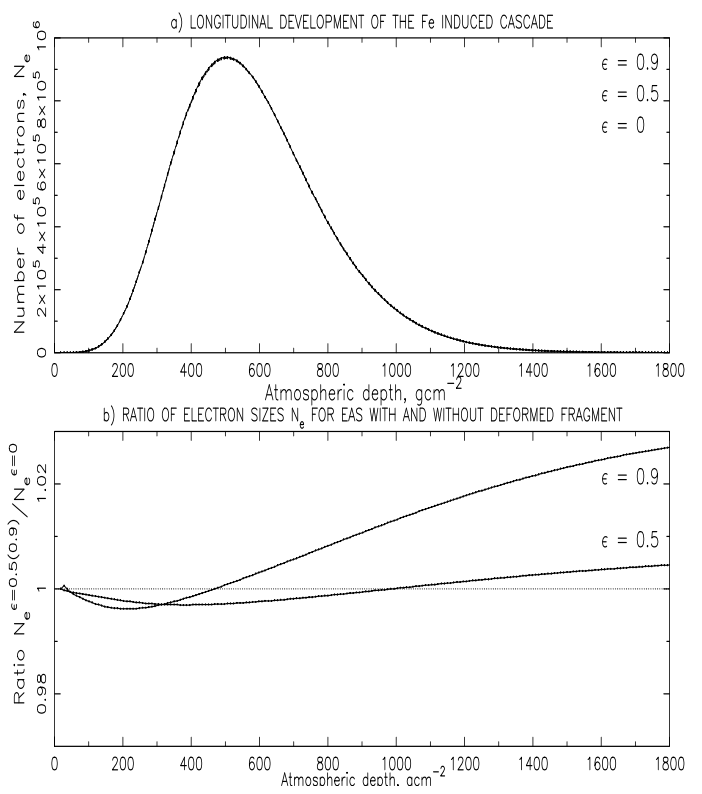

Figure 9. a) The longitudinal development of 1 PeV Feinduced EAS with the emission of the Sc fragment with the different ellipticity. The difference between $\epsilon=0,0.5$ and 0.9 is small and not seen at this graph. b) The ratio of the shower size $N_{e}$ for $\epsilon=0.5,0.9$ and 0 as the function of the atmospheric depth.

be rather small. The shift of the cascade maximum does not exceed $1 \mathrm{gcm}^{-2}$, the increase of the shower size at the sea level is about $2 \%$. These changes alone are not sufficient to eliminate inconsistencies in the interpretation of experimental data on EAS discussed in the Introduction. However, numerical values of the mass, multiplicity and ellipticity of the fragment in the examined example were taken from the experiments at $\mathrm{GeV}$ and $\mathrm{TeV}$ energies. If there is an energy dependence of these characteristics and the ellipticity rises with energy - a not unlikely situation - the sling effect can be much stronger. The elongation rate for nucleus-induced cascades will be higher than without the sling effect and the interpretation of experimental data on $X_{\max }$ distribution at ultra high energies should be modified towards a higher abundance of heavy nuclei in the mass 
composition of $\mathrm{PeV}$ and $\mathrm{EeV}$ cosmic rays. At the highest energies $\left(\simeq 10^{20} \mathrm{eV}\right)$ the effect could be very large and the consequences for the mass composition of the important extragalactic particles rather profound.

\section{Conclusion}

We have examined the possible slowing down of the development of the atmospheric cascade initiated by a primary nucleus of high energy due to a sling effect, which is the rotation, deformation and polarization of nuclear fragments emitted in nucleus-nucleus interactions. For an examined example of Fe-induced cascade, in which deformed and polarized fragment of ${ }^{45} \mathrm{Sc}$ is emitted in the very first collision of the projectile the effect has been shown to be small. However, we also showed that the effect grows with an increasing deformation. If the deformation increases with the collision energy, this effect becomes important and should be taken into account in the models of nucleus-nucleus interactions used for the simulation of the development of atmospheric cascades.

Acknowledgements One of the authors (ADE) thanks The Royal Society for financial support.

\section{REFERENCES}

1. Heck D. et al., 1998, FZKA Report Forschungszentrum Karlsruhe 6019

2. Erlykin A.D., Wolfendale A.W., 1998, Astroparticle Physics, 9, 213

3. Antoni T. et al. 1999, J.Phys.G: Nucl.Part.Phys., 25 , 2161

4. Roth M. et al. 2001, Proc. 27th ICRC, Hamburg, 1, 88

5. Haungs A. et al. 2003, Progr. Nucl. Part. Phys., 66, 1145

6. Hörandel J.R. 2003, J.Phys.G: Nucl.Part.Phys., 29, 2439

7. Yakovlev V.I. 2003, Nucl.Phys.B (Proc.Suppl.), 122, 417

8. Erlykin A.D., Wolfendale A.W., 2002, Astroparticle Physics, 18, 151
9. Waddington C.J. et al. 1990, Proc. 21st ICRC, Adelaide, 8, 87

10. Burnett T. et al. 1987, Phys. Rev. D35, 824

11. Klein S.R., 2003, Nucl.Phys.B ( Proc.Suppl.), 122, 76

12. Adler C. et al. nucl-ex/0206004

13. Satchler G.R., Introduction to Nuclear Reactions, Macmillan Press Ltd., New York, 1980

14. Dremin I.M., Man'ko V.I. 1998, Nuovo Cimento, 111A, 439

15. Bradt H.L., Peters B., 1948, 74, 1828

16. Bialas A. et al., 1976, Nucl. Phys. B111, 461

17. Shabelsky Yu.M., 1979, Acta Phys. Polonica, B10, 1049

18. Catalano O. et al, 2001, Proc. 27th ICRC, Hamburg, 2, 498 